\title{
NASA-TM-83443
}

NASA Technical Memorandum 83443

\section{Convergence and Stability Properties of Minimal Polynominal and Reduced Rank Extrapolation Algorithms}

Avram Sidi

Lewis Research Center

Cleveland, Ohio

July 1983
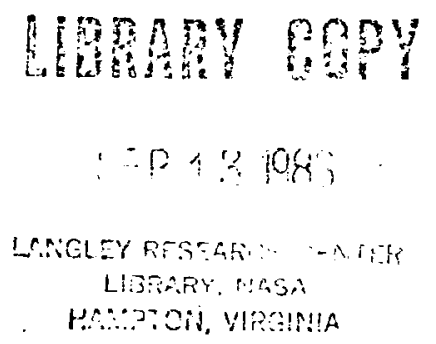


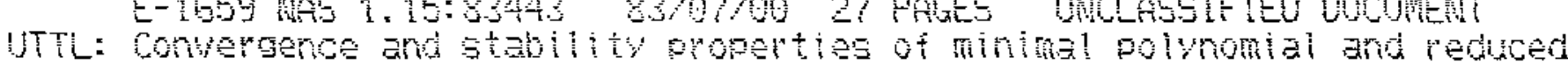

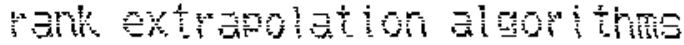

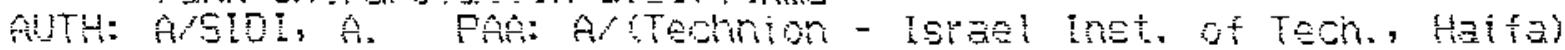

cop: He

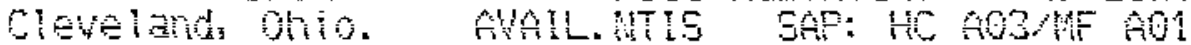

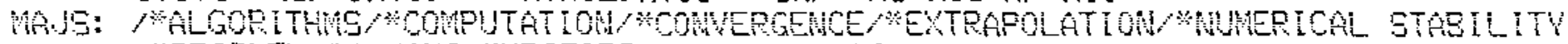
*PROBLE SOLUMG WEOTORS MATHEMTICS:

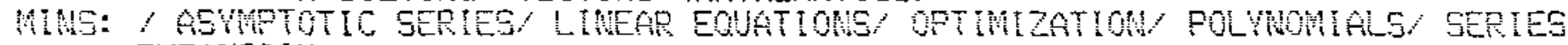
Expherou

PBA: T.G.

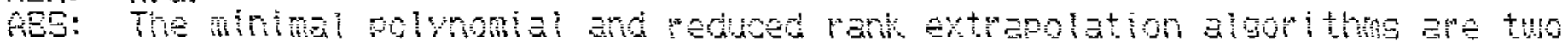
acteleration of conergene methode for seduences of rectors. In z pecont.

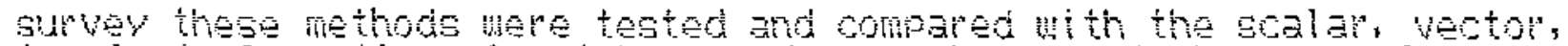

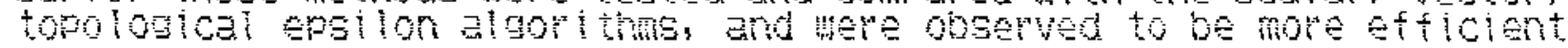

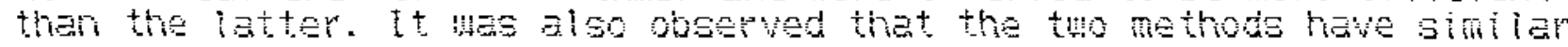
converence propties. The onvergence and otablity propeties of these

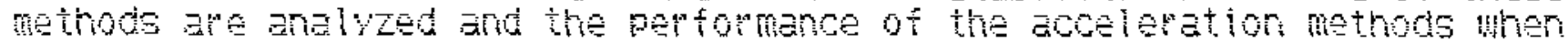
apolied to a class gt vecton secuences that iruludes those actuences

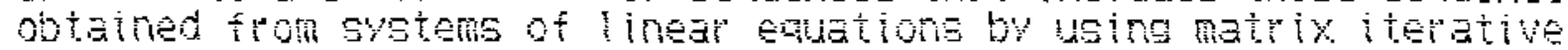
EMTE: methods is discussed. 
CONVERGENCE AND STABILITY PROPERTIES OF MINIMAL POLYNOMIAL

AND REDUCED RANK EXTRAPOLATION ALGORITHMS

\author{
Avram Sidi* \\ National Aeronautics and Space Administration \\ Lewis Research Center \\ Cleveland, Ohio 44135
}

ABSTRACT

The minimal polynomial and reduced rank extrapolation algorithms are two acceleration of convergence methods for sequences of vectors. In a recent survey these methods were tested and compared with the scalar, vector, and topological epsilon algorithms, and were observed to be more efficient than the latter. It was also observed that the two methods have similar convergence properties. The purpose of the present work is to analyze the convergence and stability properties of these methods, and to show that they are bona fide acceleration methods when applied to a class of vector sequences that includes those sequences obtained from systems of linear equations by using matrix iterative methods.

\title{
1. INTRODUCTION
}

The minimal polynomial extrapolation (MPE) and the reduced rank extrapolation (RRE) algorithms are two methods that have been devised for accelerating the convergence of sequences of vectors. In a recent survey carried out by D. A. Smith, W. F. Ford, and A. Sidi (unpublished) these two methods were tested and compared with the scalar, vector, and topological epsilon algorithms. It was observed numerically that the MPE and the RRE have similar convergence properties and are more efficient than the three epsilon algorithms. In the present work we analyze the convergence and stability properties of the

*Computer Science Department, Technion - Israel Institute of Technology, Haifa, Israel. Work partly done as a National Research Council - NASA Research Associate. 
MPE and the RRE, and show that they are bona tide convergence acceleration methods when applied to a family of sequences that includes those sequences obtained from systems of linear equations by using matrix iterative methods.

In a recent work [3] a general framework for deriving convergence acceleration methods for vector sequences has been proposed. Within this framework one can derive several methods, some old (including the MPE, RRE, and the topological epsilon algorithm), and some new (including a method that has been designated the modified MPE). The approach of [3] is formulated in general normed linear spaces of finite or infinite dimension. In the present work we use the formulations of the MPE and the RRE as they are given in [3]. We also make the assumption that the normed linear space in which the vector sequence is defined is an inner product space, with the norm being induced by the inner product.

The plan of the present paper is as follows: In Section 2 we specify the vector sequences whose convergence we are seeking to accelerate, describe the MPE and the RRE as they were formulated in [3], deriving at the same time determinant representations for them. We shall use Section 2 to also introduce much of the notation that we use in the remainder of this work. In Section 3 we analyze the convergence properties of both methods and obtain actual rates of acceleration for them. In Section 4 we analyze their stability properties. The results of Sections 3 and 4 are helpful in explaining some of the numerical results obtained from the MPE and the RRE. The techniques used in the present work are similar in nature to those developed and used in [3] in the analysis of the modified MPE and the topological epsilon algorithm. The analysis in the present work, however, is considerably heavier due to the extreme nonlinearity of the MPE and the RRE. Surprisingly, all the conclusions that were drawn for the modified MPE hold for MPE and RRE. 


\section{NOTATION AND DESCRIPTION OF ALGORITHMS}

Let $B$ be an inner product space defined over the field of complex numbers. In this work we shall adopt the following convention for the homogeneity property of the inner product. For $y, z \in B$ and $\alpha, \beta$ complex numbers, the inner product $(\cdot, \bullet)$ is defined such that $(\alpha y, \beta z)=\bar{\alpha} \beta(y, z)$. The norm of a vector $x \in B$ will be defined by $\|x\|=\sqrt{(x, x)}$.

Let us consider a sequence of vectors $x_{i}, i=0,1, \ldots$, in $B$. We shall assume that

$$
x_{m} \sim s+\sum_{i=1}^{\infty} v_{i} \lambda_{i}^{m} \text { as } m \rightarrow \infty
$$

where $s$ and $v_{i}, i=1,2, \ldots$, are vectors in $B$, and $\lambda_{i}, i=1,2, \ldots$, are scalars, such that $\lambda_{i} \neq 1, i=1,2, \ldots, \lambda_{i} \neq \lambda_{j}$ if $i \neq j,\left|\lambda_{1}\right| \geq\left|\lambda_{2}\right|$ $\geq \ldots$, and that there can be only a finite number of $\lambda_{i}$ whose moduli are equal. Without loss of generality, we assume in (2.1) that $v_{j} \neq 0, \lambda_{i} \neq 0$ for all $i \geq 0$. The meaning of $(2.1)$ is that for any integer $N>0$, there exist a positive constant $K$ and a positive integer $m_{0}$ that depend only on $N$, such that for every $m \geq m_{0}$ the vector

$$
\tilde{v}_{N}(m)=\left(x_{m}-s-\sum_{i=1}^{N-1} v_{i} \lambda_{i}^{m}\right) / \lambda_{N}^{m}
$$

satisfies

$$
\left\|\tilde{v}_{N}(m)\right\| \leq K
$$

If $\left|\lambda_{1}\right|<1$, then $\lim _{m \rightarrow \infty} x_{m}$ exists and is simply s. If $\left|\lambda_{1}\right| \geq 1$, then $\lim _{m \rightarrow \infty} x_{m}$ does not exist, and $s$ is said to be the anti-limit of the sequence $x_{m}, m=0,1, \ldots$ Our problem is to find a good approximation to $s$, whether it be the limit or the anti-limit of the sequence, from a relatively small number of the vectors $x_{i}, i=0,1, \ldots$ 
Example. Let $A$ be a nondefective MxM (complex) matrix and $b$, an M-dimensional (complex) vector, and consider the solution of the linear system of equations

$$
x=A x+b .
$$

Let $\lambda_{1}, \ldots, \lambda_{M}$ and $v_{1}, \ldots, v_{M}$ be the eigenvalues and corresponding eigenvectors of $A$. Assume also that 1 is not an eigenvalue of $A$ so that (2.2) has a unique solution, which we shall denote by $s$. For a given $x_{0}$, we generate the sequence $x_{j}, j=1,2, \ldots$, by the matrix iterative method

$$
x_{j+1}=A x_{j}+b, j=0,1, \ldots .
$$

Let $x_{0}-s=\sum_{i=1}^{M} \alpha_{j} v_{i}$ for some scalars $\alpha_{i}$. Then

$$
x_{m}=s+\sum_{i=1}^{M} \alpha_{i} v_{i} \lambda_{i}^{m}, m=0,1, \ldots .
$$

As is known if $\alpha_{1} \neq 0$, which will be the case for the given $x_{0}$ in general, then $\lim _{m+\infty} x_{m}=s$ provided $\left|\lambda_{1}\right|<1$, otherwise $s$ is the anti-limit.

Let us denote $u_{i}=\Delta x_{i}=x_{j+1}-x_{i}, i=0 ; 1, \ldots$, and $w_{i}=\Delta u_{i}=\Delta x_{i}$, $i=0,1, \ldots$.

The MPE. Let $k$ be an integer less than or equal to the dimension of the space $B$. The approximation $s_{n, k}$ to $s$ is given by

$$
s_{n, k}=\sum_{j=0}^{k} \gamma_{j} x_{n+j},
$$

where the $r_{j}$ are obtained from 


$$
r_{j}=\frac{c_{j}}{\sum_{j=0}^{k} c_{i}}, j=0,1, \ldots, k,
$$

with $c_{k}=1$, and $c_{0}, \ldots, c_{k-1}$ being determined as the solution to the minimization problem

$$
c_{0}, \ldots, c_{k-1}\left\|\sum_{j=0}^{k-1} c_{j} u_{n+j}+u_{n+k}\right\|
$$

provided $\sum_{i=0}^{k} c_{i} \neq 0$

For the example above, (2.7) is equivalent to the least squares solution of the overdetermined system of $M$ equations

$$
u_{n, k} c=-u_{n+k} \text {, }
$$

where $U_{n, k}$ is the $M \times k$ matrix

$$
u_{n, k}=\left(u_{n}, u_{n+1}, \ldots, u_{n+k-1}\right),
$$

and $c$ is the column vector $\left(c_{0}, c_{1}, \ldots, c_{k-1}\right)^{\top}$, when $(y, z)=y^{\star} z=\sum_{i=1}^{M} \bar{y}^{i} z^{i}$, where $y=\left(y^{1}, \ldots, y^{M}\right)^{\top}$ and $z=\left(z^{1}, \ldots, z^{M}\right)^{\top}$, and $y^{\star}$ is the Hermitean conjugate of $y$. This is the way the MPE was developed originally in [1]. We shall now give a determinant expression for $s_{n, k}$ that will be of use in the remainder of this work. Since $\|y\|=\sqrt{(y, y)}$ for any vector $y$ in $B$, the $c_{j}$ that solve the minimization problem in (2.7) satisfy the normal equations 


$$
\sum_{j=0}^{k-1}\left(u_{n+j}, u_{n+j}\right) c_{j}=-\left(u_{n+i}, u_{n+k}\right), 0 \leq i \leq k-1 .
$$

Consequently, the $\gamma_{j}$ that are defined by (2.6) satisfy the equations

$$
\left\{\begin{array}{l}
\sum_{j=0}^{k} \gamma_{j}=1 \\
\sum_{j=0}^{k}\left(u_{n+j}, u_{n+j}\right) \gamma_{j}=0, \quad 0 \leq i \leq k-1,
\end{array}\right.
$$

provided these equations have a solution. Assuming that the determinant of the matrix of equations (2.11) is nonzero, and using Cramer's rule, we can write the solution of (2.11) as

$$
\gamma_{j}=\frac{N_{j}}{\sum_{i=0}^{k} N_{i}}=\frac{N_{j}}{D(1, \ldots, 1)}, 0 \leq j \leq k,
$$

where $N_{j}$ is the cofator of $\sigma_{j}$ in the determinant

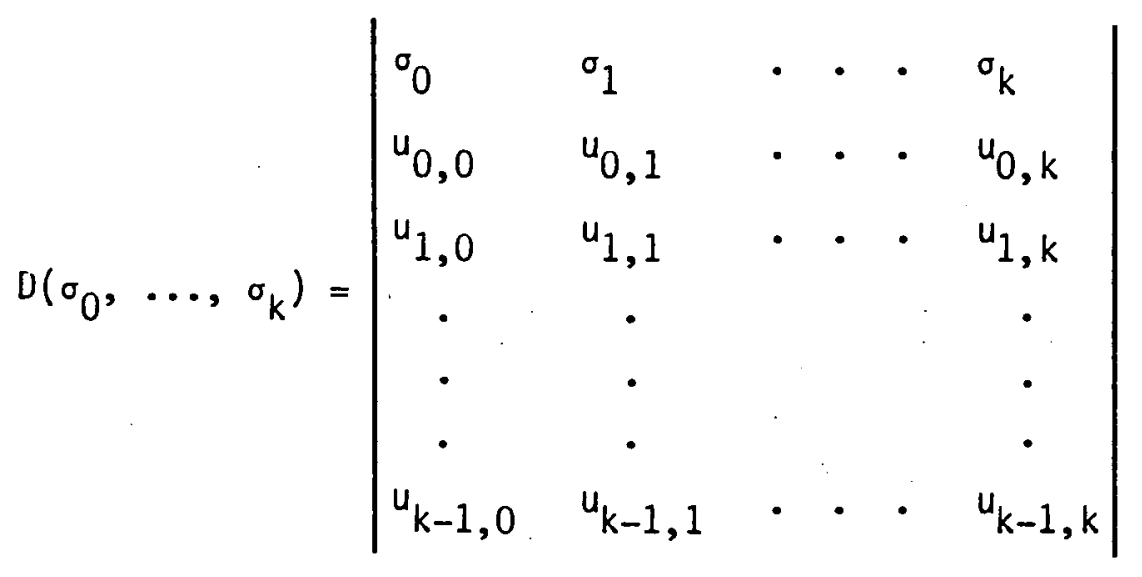

with $u_{i, j} \equiv\left(u_{n+j}, u_{n+j}\right), i, j \geq 0$. In the first row of the determinant $D\left(\sigma_{0}, \ldots, \sigma_{k}\right)$ we also allow $\sigma_{0}, \ldots, \sigma_{k}$ to be vectors in $B$, in which case (2.13) is to be interpreted as 


$$
D\left(\sigma_{0}, \ldots, \sigma_{k}\right)=\sum_{i=0}^{k} \sigma_{i} N_{i} .
$$

Combining (2.5), (2.12), and (2.14), we can now express $s_{n, k}$ as

$$
s_{n, k}=\frac{D\left(x_{n}, \cdots, x_{n+k}\right)}{D(1, \cdots, 1)}
$$

The RRE. Let $k$ be an integer less than or equal to the dimension of the space $B$. The approximation $s_{n, k}$ to $s$ is given by

$$
s_{n, k}=x_{n}+\sum_{j=0}^{k-1} q_{j} u_{n+i}
$$

where the $q_{j}$ are determined as the solution of the minimization problem

$$
q_{0}, \ldots i n q_{k-1}\left\|u_{n}+\sum_{i=0}^{k-1} q_{i} w_{n+i}\right\|
$$

For the example above, (2.17) is equivalent to the least squares solution of the overdetermined system of equations

$$
w_{n, k^{q}}=-u_{n} \text {, }
$$

where $W_{n, k}$ is the $M \times k$ matrix

$$
w_{n, k}=\left(w_{n}, w_{n+1}, \ldots, w_{n+k-1}\right) \text {, }
$$

and $q$ is the column vector $q=\left(q_{0}, q_{1}, \ldots, q_{k-1}\right)^{\top}$, when $(y, z)=\sum_{i=1}^{M} \bar{y}_{i} z_{i}$,

where $y$ and $z$ are again $y=\left(y^{1}, \ldots, y^{M}\right)^{\top}$ and $z=\left(z^{1}, \ldots, z^{M}\right)^{\top}$. This is the way the RRE was developed originally in [2]. 
For this case too $s_{n, k}$ can be expressed as the quotient of two determinants, and we turn to this now. Again by the fact that $\|y\|=\sqrt{(y, y)}$ for any vector $y$ in $B$, the $q_{j}$ that solve the minimization problem in (2.17) satisfy the normal equations

$$
\sum_{j=0}^{k-1}\left(w_{n+j}, w_{n+j}\right) q_{j}=-\left(w_{n+j}, u_{n}\right), 0 \leq i \leq k-1 .
$$

Substituting $w_{n+j}=u_{n+j+1}-u_{n+j}$ on the left hand side of (2.20), and rearranging, we obtain

$$
\begin{array}{r}
\left(w_{n+j}, u_{n}\right)\left(1-q_{0}\right)+\sum_{j=1}^{k-1}\left(w_{n+i}, u_{n+j}\right)\left(q_{j-1}-q_{j}\right)+\left(w_{n+j}, u_{n+k}\right) q_{k-1}=0 \\
0 \leq i \leq k-1 .
\end{array}
$$

Let us define

$$
\gamma_{0}=1-q_{0}, \gamma_{k}=q_{k-1}, \gamma_{j}=q_{j-1}-q_{j}, 1 \leq j \leq k-1 \text {. }
$$

It is easily verified that

$$
\sum_{j=0}^{k} \gamma_{j}=1
$$

so that (2.22) and (2.23) establish a one-to-one correspondence between the $q_{j}, 0 \leq i \leq k-1$, and the $\gamma_{j}, 0 \leq j \leq k$. Consequently, the linear system of equations (2.20) for the $q_{j}$ is equivalent to the linear system

$(2.24)$

$$
\left\{\begin{array}{l}
\sum_{j=0}^{k} r_{j}=1 \\
\sum_{j=0}^{k}\left(w_{n+j}, u_{n+j}\right) r_{j}=0,0 \leq i \leq k-1,
\end{array}\right.
$$


for the $\gamma_{j}$, by (2.21), (2.22), and (2.23). Similarly, substituting $u_{n+i}=x_{n+i+1}-x_{n+i}$ on the right hand side of (2.16), rearranging, and invoking (2.22), we see that $s_{n, k}$ for RRE (as for MPE) can be expressed in the form (2.5): Assuming now that the determinant of the matrix of equations (2.24) is nonzero, and using Cramer's rule, we can express the $\gamma_{j}$ that solve (2.24) exactly as in (2.12), where $N_{j}$ is the cofactor of $\sigma_{j}$ in the determinant $D\left(\sigma_{0}, \ldots, \sigma_{k}\right)$ given in (2.13) with $u_{i, j} \equiv\left(w_{n+i}, u_{n+j}\right), i, j \geq 0$. Again when $\sigma_{0}, \ldots, \sigma_{k}$ are vectors in $B, D\left(\sigma_{0}, \ldots, \sigma_{k}\right)$ is to be interpreted as in (2.14). Consequently $s_{n, k}$, also for RRE, can be expressed as in (2.15). Before closing this section we shall state a result that will be of use in the remainder of this work.

Lemma 2.1. Let $i_{0}, i_{1}, \ldots, i_{k}$ be integers greater than or equal to 1 , and assume that the scalars $v_{i_{0}}, \ldots, i_{k}$ are odd under an interchange of any two indices $i_{0}, \ldots, i_{k}$. Let $\sigma_{i}, i \geq 1$, be scalars (or vectors) and let $t_{i, j}, i \geq 1,1 \leq j \leq k$, be scalars. Define

$$
I_{k, N}=\sum_{i_{0}=1}^{N} \cdots \sum_{i_{k}=1}^{N} \sigma_{i_{0}}\left(\prod_{p=1}^{k} t_{i_{p}, p}\right) v_{i_{0}, \ldots, i_{k}}
$$

and

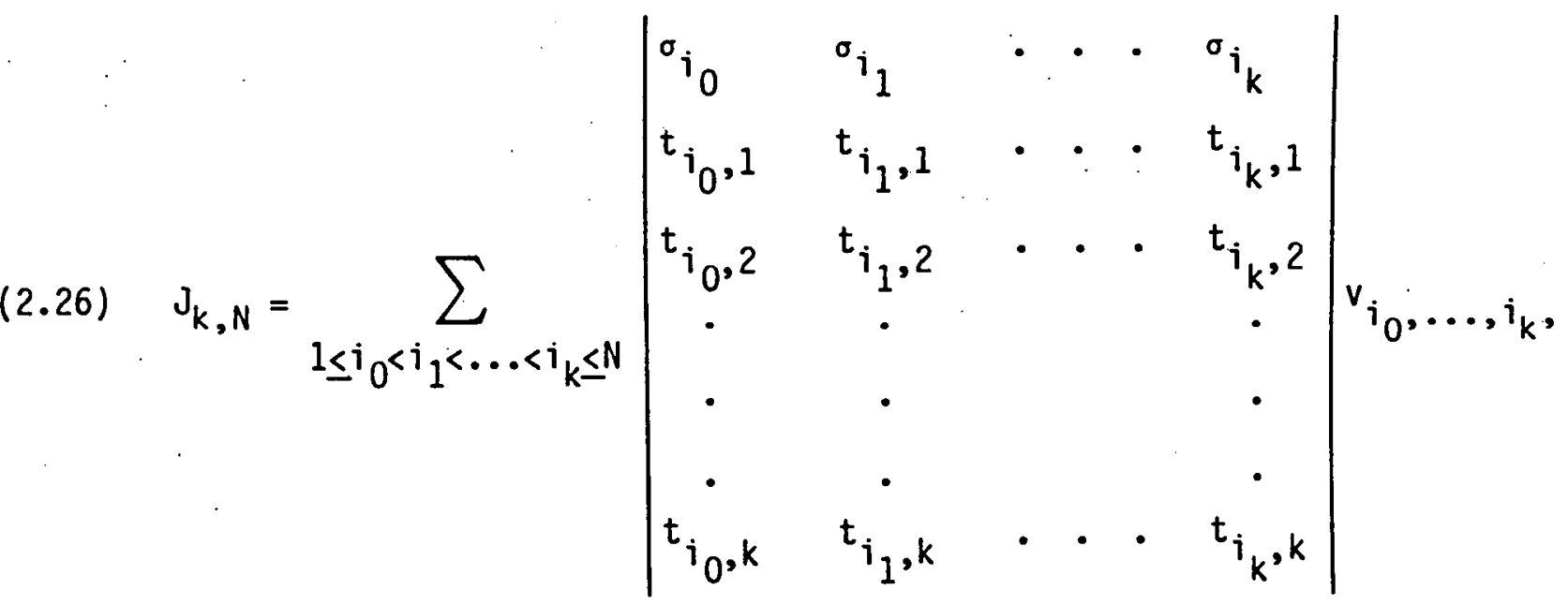


where the determinant in (2.26) is to be interpreted in the same way as $D\left(\sigma_{0}, \ldots, \sigma_{k}\right)$ in $(2.13)$. Then

$$
I_{k, N}=J_{k, N}
$$

For a proof of Lemma 2.1, see [3, Appendix].

\section{CONVERGENCE ANALYSIS}

We have seen in the previous section that $s_{n, k}$, for both the MPE and the RRE, is given by

$$
s_{n, k}=\frac{D\left(x_{n}, \ldots, x_{n+k}\right)}{D(1, \ldots, 1)}
$$

where $D\left(\sigma_{0}, \ldots, \sigma_{k}\right)$ is defined by (2.13) with $u_{i, j}=\left(u_{n+i}, u_{n+j}\right), i, j \geq 0$, for MPE, and $u_{i, j}=\left(w_{n+i}, u_{n+j}\right), i, j \geq 0$, for RRE. Subtracting $s$ from both sides of (3.1), and making use of (2.14), we obtain the error formula

$$
s_{n, k}-s=\frac{D\left(x_{n}-s, \ldots, x_{n+k}-s\right)}{D(1, \ldots, 1)}
$$

Under the assumption (2.1), we have

$$
u_{m} \sim \sum_{i=1}^{\infty} v_{i}\left(\lambda_{i}-1\right) \lambda_{i}^{m} \text { as } m+\infty \text {, }
$$

and consequently,

$$
w_{m} \sim \sum_{i=1}^{\infty} v_{i}\left(\lambda_{i}-1\right)^{2} \lambda_{j}^{m} \text { as } m+\infty
$$

From (3.3) and (3.4) we see that 
(3.5) $\quad u_{p, q}=\left(u_{n+p}, u_{n+q}\right) \sim \sum_{i=1}^{\infty} \sum_{j=1}^{\infty}\left(v_{i}, v_{j}\right)\left(\bar{\lambda}_{i}-1\right)\left(\lambda_{j}-1\right) \lambda_{i}^{n+p} \lambda_{j}^{n+q}$

as $n \rightarrow \infty$,

for MPE, and

$$
u_{p, q}=\left(w_{n+p}, u_{n+q}\right)-\sum_{j=1}^{\infty} \sum_{j=1}^{\infty}\left(v_{j}, v_{j}\right)\left(\bar{\lambda}_{i}-1\right)^{2}\left(\lambda_{j}-1\right) \bar{\lambda}_{i}{ }^{n+p} \lambda_{j}^{n+q}
$$

for RRE. (3.5) and (3.6) can be rewritten in the condensed and unified form

$$
u_{p, q} \sim \sum_{i=1}^{\infty} \sum_{j=1}^{\infty} z_{i j} \bar{\lambda}_{i}^{n+p} \lambda_{j}^{n+q} \text { as } n+\infty
$$

where we have defined

$$
z_{i j}=\left(v_{i}, v_{j}\right)\left(\bar{\lambda}_{i}-1\right)\left(\lambda_{j}-1\right), i, j \geq 1,
$$

for MPE, and

$$
z_{i j}=\left(v_{i}, v_{j}\right)\left(\bar{\lambda}_{i}-1\right)^{2}\left(\lambda_{j}-1\right), i, j \geq 1,
$$

for RRE.

Note that when the sequence $x_{j}, j=0,1, \ldots$, is generated by a matrix iterative process as described in the example of Section 2, then in (3.7) the upper limits on the summations on the right hand side are replaced by $M$, and $\sim$ is replaced by $=$.

In Lemma 3.1 and Lemma 3.2 below we derive the asymptotic expansions of $D\left(x_{n}-s, \ldots, x_{n+k}-s\right)$ and $D(1, \ldots, 1)$, respectively, for $n+\infty$, assuming (2.1). In Theorem 3.1 we give the main result of this paper that shows that 
both the MPE and RRE are true acceleration methods when applied to sequences of vectors satisfying (2.1), in the sense that $\lim _{n \rightarrow \infty}\left\|s_{n, k}-s\right\| /\left\|x_{n+k+1}-s\right\|=0$.

In what follows we shall denote asymptotic relations of the form " $\alpha_{n} \sim B_{n}$ as $n_{+}+\infty$ " by " $\alpha_{n} \sim \beta_{n}$ " for short. Also we shall let $\sum_{i} \equiv \sum_{i=1}^{\infty}$, $\sum_{i, j} \equiv \sum_{i=1}^{\infty} \sum_{j=1}^{\infty}$, and $\sum_{1 \leq i_{1}<i_{2}<\ldots<i_{k}} \equiv \sum_{i_{1}=1}^{\infty} \sum_{i_{2}=i_{1}+1}^{\infty} \cdots \sum_{i_{k}=i_{k-1}+1}^{\infty}$, for simplicity.

Lemma 3.1. Let the sequence of vectors $x_{i}, i=0,1,2, \ldots$, be as described in the previous section. For $i_{p}, j_{p}$ positive integers, define

$$
R_{j_{0}, j_{1}, \ldots, j_{k}}^{i_{1}, \ldots, i_{k}}=\left|\begin{array}{ccccc}
v_{j_{0}} & v_{j_{1}} & \cdot & \cdot & v_{j_{k}} \\
z_{i_{1} j_{0}} & z_{i_{1} j_{1}} & \cdot & \cdot & z_{i_{1} j_{k}} \\
z_{i_{2} j_{0}} & z_{i_{2} j_{1}} & \cdot & \cdot & z_{i_{2} j_{k}} \\
\cdot & \cdot & & & \cdot \\
\cdot & \cdot & & & \cdot \\
\cdot & \cdot & & & \cdot \\
z_{i_{k} j_{0}} & z_{i_{k} j_{1}} & \cdot & \cdot & z_{i_{k} j_{k}}
\end{array}\right|,
$$

where the interpretation of this determinant is like that of $D\left(\sigma_{0}, \sigma_{1}, \ldots, \sigma_{k}\right)$ in (2.13) and $(2.14)$. Let $V\left(\xi_{0}, \xi_{1}, \ldots, \xi_{k}\right)$ be the Vandermonde determinant

$$
V\left(\xi_{0}, \xi_{1}, \ldots, \xi_{k}\right)=\left|\begin{array}{ccccc}
1 & \xi_{0} & . & . & \xi_{0}^{k} \\
1 & \xi_{1} & \cdot & \cdot & \xi_{1}^{k} \\
\cdot & \cdot & & & \cdot \\
\cdot & \cdot & & & \cdot \\
\cdot & \cdot & & & \dot{k} \\
1 & \xi_{k} & . & \cdot & \xi_{k}^{k}
\end{array}\right|=\prod_{0 \leq i<j \leq k}\left(\xi_{j}-\xi_{j}\right) .
$$

Then $D\left(x_{n}-s, \ldots, x_{n+k}-s\right)$ has the asymptotic expansion given by 


$$
\begin{aligned}
& D\left(x_{n}-s, \ldots, x_{n+k}-s\right) \sim \sum_{1 \leq j_{0}<j_{1}<\ldots<j_{k}}\left(\prod_{p=0}^{k} \lambda_{j_{p}}^{n}\right) \\
& x v\left(\lambda_{j_{0}}, \lambda_{j_{1}}, \ldots, \lambda_{j_{k}}\right) \sum_{1 \leq i_{1}<i_{2}<\ldots<i_{k}}\left(\prod_{p=1}^{k} \bar{\lambda}_{i_{p}}^{n}\right) \\
& \quad \times v\left(\bar{\lambda}_{i_{1}}, \ldots, \bar{\lambda}_{i_{k}}\right) R_{j_{0}, j_{1}, \ldots, j_{k}}^{i_{1}, \ldots} .
\end{aligned}
$$

Proof. Let us denote $D\left(x_{n}-s, \ldots, x_{n+k}-s\right)$ by $P_{n}$ for short. By (2.13), (2.1), and (3.7), we have

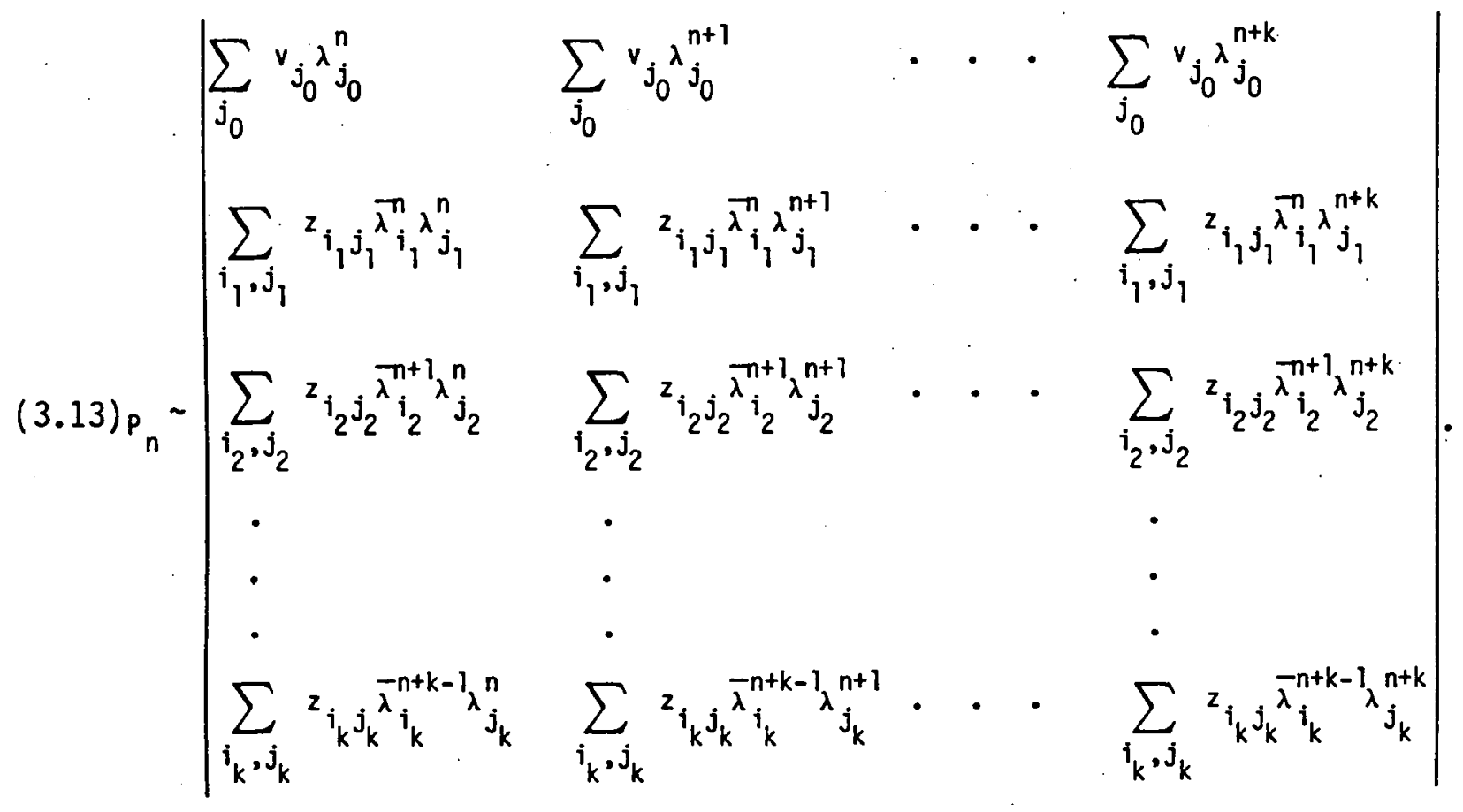

By the multilinearity property of determinants we can reexpress (3.13) in the form 


$$
\begin{array}{r}
P_{n}-\sum_{j_{0}} \sum_{i_{1}, j_{1}} \cdot \sum_{i_{k}, j_{k}} v_{j_{0}}{ }^{n}{ }_{j_{0}}\left(\prod_{p=1}^{k} z_{j_{p}} \vec{j}_{p}^{\lambda_{i}^{n+p-1} \lambda_{j}^{n}}\right) \\
\quad x v\left(\lambda_{j_{0}}, \lambda_{j_{1}}, \ldots, \lambda_{j_{k}}\right)
\end{array}
$$

By changing the order of summation in (3.14), we have

$$
\begin{aligned}
P_{n} \sim \sum_{i_{1}} \cdot \sum_{i_{k}}\left(\prod_{p=1}^{k} \lambda_{i_{p}}^{n+p-1}\right) & \sum_{j_{0}} \sum_{j_{1}} \cdot \sum_{j_{k}} v_{j_{0}}\left(\prod_{p=1}^{k} z_{i_{p} j_{p}}\right) \\
& \times\left(\prod_{p=0}^{k} \lambda_{j_{p}}^{n}\right) v\left(\lambda_{j_{0}}, \lambda_{j_{1}}, \ldots, \lambda_{j_{k}}\right) .
\end{aligned}
$$

Since $\left(\prod_{p=0}^{k} \lambda_{j_{p}}^{n}\right) \vee\left(\lambda_{j_{0}}, \lambda_{j_{l}}, \ldots, \lambda_{j_{k}}\right)$ is odd under an interchange of any two of the indices $j_{p}$, Lemma 2.1 can be applied, and we obtain

$$
\begin{aligned}
& P_{n}-\sum_{1_{1}} \cdot \sum_{i_{k}}\left(\prod_{p=1}^{k} \bar{\lambda}_{i}^{n+p-1}\right) \sum_{1 \leq j_{0}<j_{1}<\ldots<j_{k}} R_{j_{0}, j_{1}, \ldots, j_{k}}^{i_{1}, \ldots, i_{k}} \\
& x\left(\prod_{p=0}^{k} \lambda_{j_{p}}^{n}\right) v\left(\lambda_{j_{0}}, \lambda_{j_{1}}, \ldots, \lambda_{j_{k}}\right)
\end{aligned}
$$

Interchanging the order of the summations in (3.16), we have 
(3.17) $\quad P_{n} \sim \sum_{1 \leq j_{0}<j j_{1}<\ldots<j_{k}}\left(\prod_{p=0}^{k} \lambda_{j_{p}}^{n}\right) v\left(\lambda_{j_{0}}, \lambda_{j_{1}}, \ldots, \lambda_{j_{k}}\right)$

$$
\times \sum_{i_{1}} \cdots \sum_{i_{k}}\left(\prod_{p=1}^{k}{ }_{i_{i}}^{-p-1}\right)\left(\prod_{p=1}^{k}{ }^{-n}{ }_{i_{p}}^{n}\right) R_{j_{0}, j_{1}}^{i_{1}, \ldots, i_{k}} \cdot
$$

Let now $N_{j_{q}}^{i_{1}, \ldots, i_{k}}$ : be the cofactor of $v_{j_{q}}$ in the determinant expression for $R_{j_{0}, j_{1}, \ldots, j_{k}}^{i_{1}, \ldots, j_{k}}$. Then

$$
R_{j_{0}, j_{1}, \ldots, j_{k}}^{i_{1}, \ldots, i_{k}}=\sum_{q=0}^{k} v_{j_{q}} N_{j_{q}}^{i_{1}, \ldots, i_{k}} .
$$

observing from (3.10) that the $N_{j_{q}}^{i}{ }^{1} \ldots i_{k}$ are odd under an interchange of any two of the indices $i_{1}, \ldots, i_{k}$, we can apply Lemma 2.1 again to the multiple sum with respect to the indices $i_{1}, \ldots, i_{k}$, with $R_{j_{0}, j_{1}, \ldots, j_{k}}^{i_{1}, \ldots i_{k}}$ replaced by (3.18). The terms that are odd in the indices $i_{1}, \ldots, i_{k}$ now are $\left(\prod_{p=1}^{k} \vec{\lambda}_{i_{p}}\right) N_{j_{q}}^{i_{1}}, \ldots, i_{k}$. By Lemma 2.1 then

$$
\begin{aligned}
& P_{n} \sim \sum_{1 \leq j_{0}<j_{1}<\ldots<j_{k}}\left(\prod_{p=0}^{k} \lambda_{j_{p}}^{n}\right) v\left(\lambda_{j_{0}}, \lambda_{j_{1}}, \ldots, \lambda_{j_{k}}\right) \\
& \quad \times \sum_{q=0}^{k} v_{j_{q}} \sum_{i_{1}} \ldots \sum_{i_{k}}\left(\prod_{p=1}^{k} \bar{\lambda}_{i_{p}}^{n}\right) N_{j_{q}}{ }^{i}, \ldots, i_{k} v\left(\bar{\lambda}_{i_{1}}, \ldots, \bar{\lambda}_{i_{k}}\right),
\end{aligned}
$$

which, by invoking (3.18), can be reexpressed as (3.12), thus completing the proof. 
Lemma 3.2. Let the sequence of vectors $x_{i}, i=0,1,2, \ldots$, be as described in the previous section. For $i_{p}, j_{p}$ positive integers, define

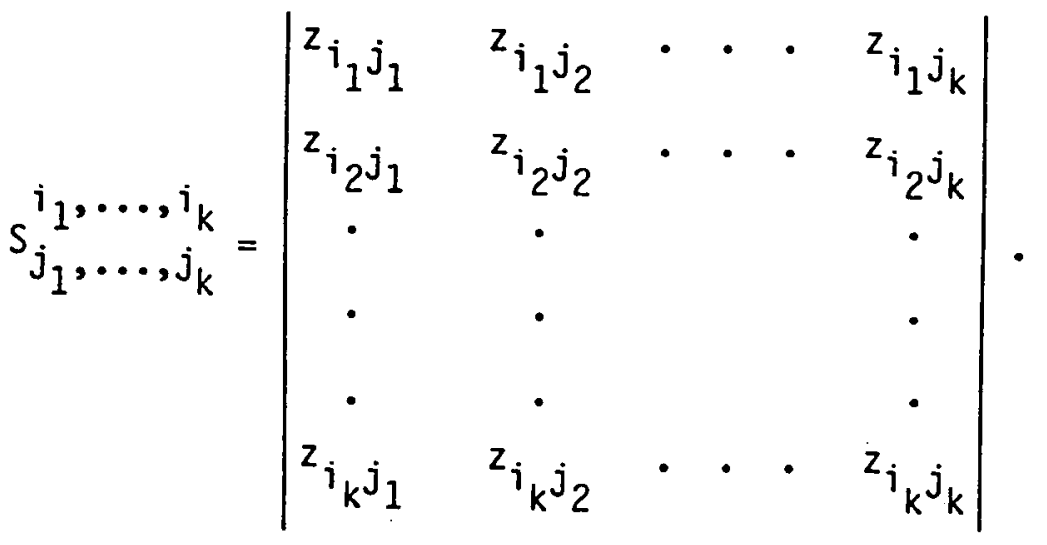

Then $D(1, \ldots, 1)$ has the asymptotic expansion given by

$$
\begin{aligned}
D(1, \ldots, 1) & \sim \sum_{1 \leq j_{1}<j_{2}<\ldots<j_{k}}\left(\prod_{p=1}^{k} \lambda_{j_{p}}^{n}\right) v\left(1, \lambda_{j_{1}}, \ldots, \lambda_{j_{k}}\right) \\
& \times \sum_{1 \leq i_{1}<i_{2}<\ldots<i_{k}}\left(\prod_{p=1}^{k} \bar{\lambda}_{i_{p}}^{n}\right) \vee\left(\bar{\lambda}_{i_{1}}, \ldots, \bar{\lambda}_{i_{k}}\right) s_{j_{1}, \ldots, j_{k}}^{j_{1}, \ldots, i_{k}} .
\end{aligned}
$$

Proof. Let us denote $D(1, \ldots, 1)$ by $Q_{n}$ for short. From (2.13) and (3.7), we have

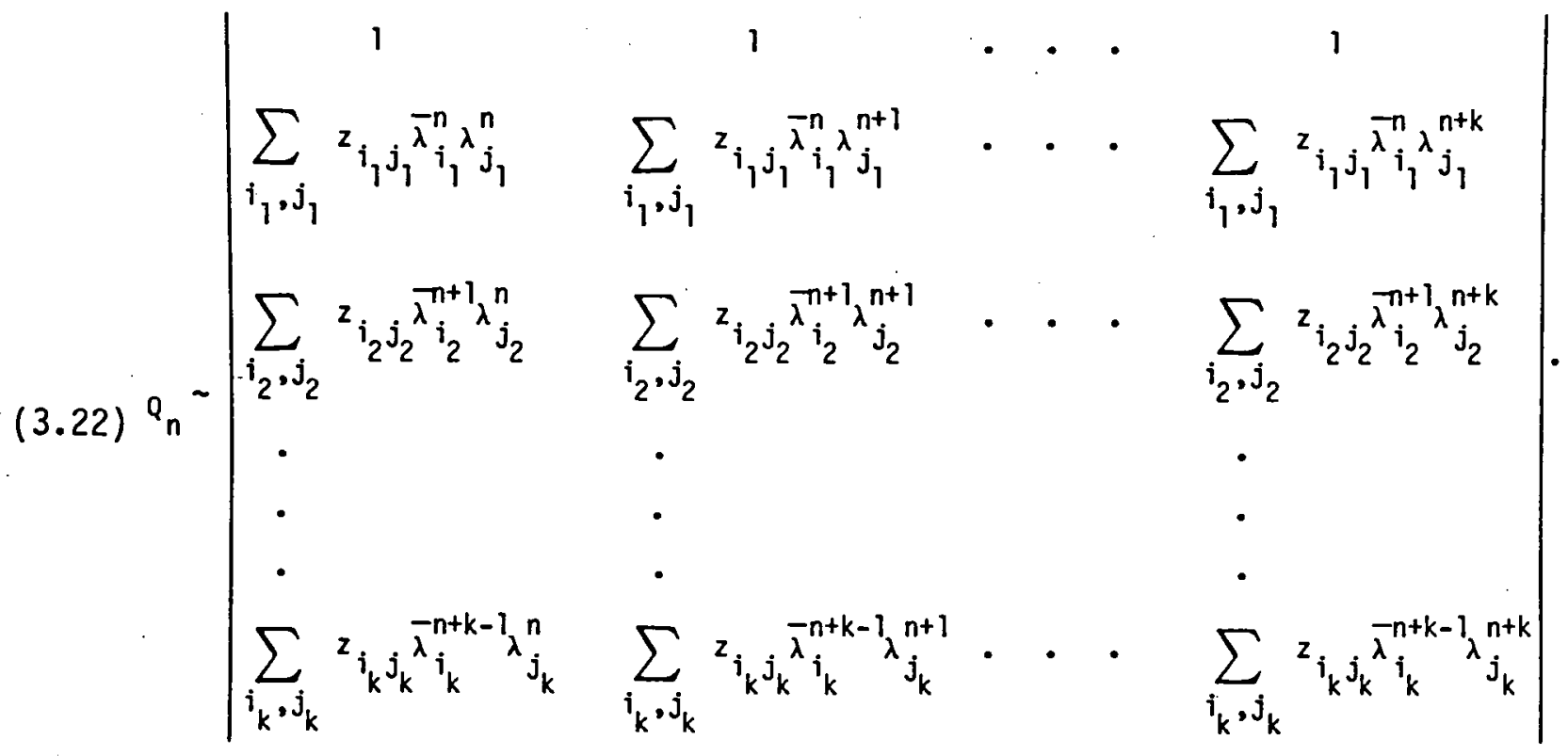


Again by the multilinearity property of determinants

$$
Q_{n} \sim \sum_{i_{1}, j_{1}} \cdots \sum_{i_{k}, j_{k}}\left(\prod_{p=1}^{k} z_{i_{p} j_{p}} \vec{\lambda}_{p}^{n+p-1} \lambda_{j_{p}}^{n}\right) v\left(1, \lambda_{j_{1}}, \ldots, \lambda_{j_{k}}\right) .
$$

By changing the order of summation in (3.23) and observing that

$\left(\prod_{p=1}^{k} \lambda_{j_{p}}^{n}\right) v\left(1, \lambda_{j_{1}}, \ldots, \lambda_{j_{k}}\right)$ is odd under an interchange of any two of the indices $j_{p}$, we can apply Lemma 2.1 to the summation over $j_{l}, \ldots, j_{k}$. The result is

$$
\begin{aligned}
& Q_{n}-\sum_{i_{1}} \cdot \sum_{i_{k}}\left(\prod_{p=1}^{k} \vec{\lambda}_{i p}^{n+p-1}\right) \\
& x \sum_{1 \leq j_{1}<j_{2}<\ldots<j_{k}} s_{j_{1}}^{i_{1}, \ldots,,_{k}}{ }_{j_{k}}\left(\prod_{p=1}^{k} \lambda_{j_{p}}^{n}\right) v\left(1, \lambda_{j_{1}}, \ldots, \lambda_{j_{k}}\right) \cdot
\end{aligned}
$$

Changing the order of summation again, and observing that $\left(\prod_{p=1}^{k} \vec{\lambda}_{i_{p}}^{n}\right) s_{j}^{i}{ }_{j}, \ldots, j_{k}$

is odd under an interchange of any two of the indices $i_{p}$, we can now apply Lemma 2.1 to the summation over $i_{1}, \ldots, i_{k}$. As a result, we obtain (3.21). This completes the proof.

Theorem 3.1. Let the sequence of vectors $x_{i}, i=0,1,2, \ldots$, be as described in the previous section. If, in addition, the $v_{i}$ are linearly independent, and

$$
\left|\lambda_{1}\right| \geq \cdots \geq\left|\lambda_{k}\right|>\left|\lambda_{k+1}\right| \geq\left|\lambda_{k+2}\right| \geq \cdots \cdot \cdot
$$


then, for all sufficiently large $n, s_{n, k}$ exists, and

$$
s_{n, k}-s=F(n) \lambda_{k+1}^{n}[1+o(1)] \text { as } n+\infty \text {, }
$$

where the vector $F(n)$ satisfies $\|F(n)\| \leq \tilde{K}$, for some constant $\tilde{K}>0$ independent of $n$, and for all sufficiently large $n$. If, in addition,

$$
\left|\lambda_{k+1}\right|>\left|\lambda_{k+2}\right|
$$

then

$$
F(n)=\frac{R_{1, \ldots, k, 1}^{1, \ldots, k}}{s_{1, \ldots, k, k}^{1, \ldots, k}} \prod_{i=1}^{k}\left(\frac{\lambda_{k+1}-\lambda_{i}}{\lambda_{i}-1}\right)[1+o(1)] \text { as } n+\infty \text {. }
$$

Proof. From (3.20) and (3.8) (or (3.9)),

$$
s_{1, \ldots, k}^{l, \ldots, k}=H G\left(v_{1}, \ldots, v_{k}\right) \text {, }
$$

where $H=\left|\prod_{i=1}^{k}\left(\lambda_{i}-1\right)\right|^{2}$ for MPE (or $H=\prod_{i=1}^{k}\left(\bar{\lambda}_{i}-1\right)^{2}\left(\lambda_{i}-1\right)$ for RRE $)$,

and $G\left(v_{1}, \ldots, v_{k}\right)$ is the Gram determinant of the vectors $v_{1}, \ldots, v_{k}$, given by

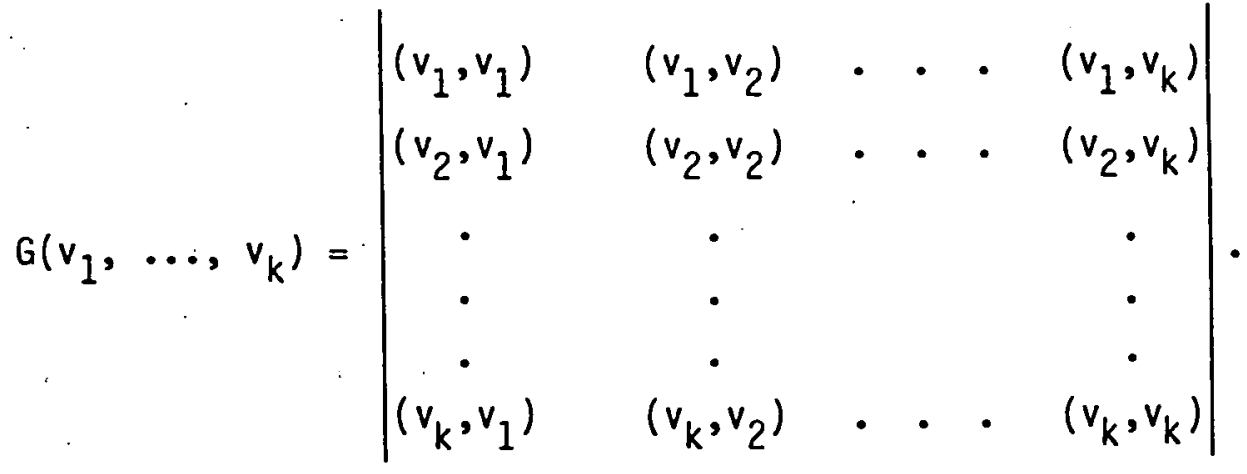

Since the $v_{j}$ are linearly independent, $G\left(v_{1}, \ldots, v_{k}\right)$ is nonzero. Also, since $\lambda_{j} \neq 1$ for all $i, H$ is nonzero. Consequently, $s_{1, \ldots, k}^{1, \ldots, k}$ is nonzero. Next, since $\lambda_{j} \neq \lambda_{j}$ for $i \neq j$, and $\lambda_{j} \neq 1$ for all $i, V\left(\bar{\lambda}_{1}, \ldots, \bar{\lambda}_{k}\right)$ and 
$V\left(1, \lambda_{1}, \ldots, \lambda_{k}\right)$ are nonzero on account of (3.11). Finally, we observe that, due to (3.25), the dominant term in the multiple sum on the right hand side of (3.21) in Lemma 3.2 would be that for which $i_{p}=p, j_{p}=p, p=1, \ldots, k$, provided $v\left(1, \lambda_{1}, \ldots, \lambda_{k}\right)$ and $s_{1}^{1, \ldots, k, k}$ are nonzero, which we have already proved to be so. Consequently,

$$
\begin{array}{r}
D(1, \ldots, 1)=\left|\prod_{p=1}^{k} \lambda_{p}^{n}\right|^{2} V\left(1, \lambda_{1}, \ldots, \lambda_{k}\right) V\left(\bar{\lambda}_{1}, \ldots, \bar{\lambda}_{k}\right) \\
\quad \times s_{1, \ldots, k}^{1, \ldots, k}[1+o(1)] \text { as } n+\infty .
\end{array}
$$

This also implies that, for all sufficiently large $n, D(1, \ldots, 1) \neq 0$, hence $s_{n, k}$ exists. This completes the proof of the first part of the theorem.

To prove (3.26), we should analyze the behavior of $D\left(x_{n}-s, \ldots\right.$,

$\left.x_{n+k}-5\right)$ for $n+\infty$. By our assumptions made following (2.1), there is only a finite number of $\lambda_{i}$ whose moduli are $\left|\lambda_{k+1}\right|$. Let $\left|\lambda_{k+1}\right|=\cdots=\left|\lambda_{k+r}\right|>$ $\left|\lambda_{k+r+1}\right|$. From this and (3.25), it follows that the dominant term on the right hand side of (3.12) - provided it is nonzero - is the sum of those terms with indices $i_{p}=p, p=1, \ldots, k, j_{p-1}=p, p=1, \ldots, k, j_{k}=k+\ell, \ell=1$, $\ldots, r$, namely

$$
\begin{aligned}
& \dot{D}\left(x_{n}-s, \ldots, x_{n+k}-s\right)=\left|\prod_{p=1}^{k} \lambda_{p}^{n}\right|^{2} v\left(\bar{\lambda}_{1}, \ldots, \bar{\lambda}_{k}\right) \\
& \quad x \sum_{\ell=1}^{r} \lambda_{k+\ell}^{n} v\left(\lambda_{1}, \ldots, \lambda_{k}, \lambda_{k+\ell}\right) R_{1}^{1}, \ldots, k, k, k+l \\
&
\end{aligned}
$$

From (3.10) and (3.20), 


$$
R_{1, \ldots, k, k+\ell}^{1, \ldots, k}=\sum_{i=1}^{k} \beta_{\ell j} v_{i}+(-1)^{k} S_{1, \ldots, k}^{1, \ldots, k} v_{k+\ell},
$$

so that the summation on the right hand side of (3.32) becomes

$$
\begin{aligned}
\sum_{i=1}^{k} v_{i}\left[\sum_{\ell=1}^{r} \lambda_{k+l}^{n} v\left(\lambda_{1}, \ldots, \lambda_{k}, \lambda_{k+\ell}\right) \beta_{\ell l}\right] & \\
& +(-1)^{k} s_{1}^{1}, \ldots, k \sum_{\ell=1}^{r} \lambda_{k+l}^{n} v\left(\lambda_{1}, \ldots, \lambda_{k}, \lambda_{k+l}\right) v_{k+l} .
\end{aligned}
$$

Since $s_{1, \ldots, k}^{1, \ldots, k} \neq 0, v\left(\lambda_{1}, \ldots, \lambda_{k}, \lambda_{k+\ell}\right) \neq 0$, and the vectors $v_{i}$ are 1 inearly independent, the second summation in (3.34) is never zero. This proves (3.32). Combining (3.31) and (3.32), (3.26) follows. If (3.27) holds, then $r=1$. In this case (3.28) follows from $(3.31),(3.32)$, and (3.11). This completes the proof of the theorem.

The asymptotic error analysis of the MPE and RRE as given in Theorem 3.1, leads us to the following important conclusions:

(1) Under the conditions stated in the theorem, the MPE and RRE are bona fide acceleration methods in the sense that

$$
\frac{\left\|s_{n, k}-s\right\|}{\left\|x_{n+k+1}-s\right\|}=0\left[\left(\frac{\lambda_{k+1}}{\lambda_{1}}\right)^{n}\right] \text { as } n+\infty .
$$

This means that if $x_{n}+s$ as $n+\infty$, i.e., $\left|\lambda_{1}\right|<1$, then $s_{n, k}+s$ as $n+\infty$, and more quickly. Also if $\lim _{m \rightarrow \infty} x_{m}$ does not exist, i.e., $\left|\lambda_{1}\right| \geq 1$, then $s_{n, k}+s$ as $n+\infty$, provided that $\left|\lambda_{k+1}\right|<1$. The reason that we write $x_{n+k+1}$ in (3.35) is that $s_{n, k}$ in both the MPE and RRE is computed from. $x_{n}, x_{n+1}, \ldots, x_{n+k+1} \cdot$ 
(2) When the MPE and RRE are being applied to a vector sequence generated by using a matrix iterative method, they will be especially effective when the iteration matrix has a small number of large eigenvalues (k-many when $s_{n, k}$ is being used) that are well separated from the small eigenvalues.

(3) As can be seen from (3.28), a loss of accuracy will take place in $s_{n, k}$ when $\lambda_{1}, \ldots, \lambda_{k}$ are close to 1 . For sequences of vectors obtained from the iterative solution of linear systems of equations, this means that the matrix of the system is nearly singular.

These conclusions are the same as those for the modified MPE, which has been defined and analyzed in [3].

Finally note that the results of this section (and of Section 4) will not change if the MPE and RRE are replaced by any other method giving rise to (3.1), with $D\left(\sigma_{0}, \ldots, \sigma_{k}\right)$ defined as in (2.13), as long as the $u_{i, j}$ in (2.13) satisfy (3.7) with $z_{i j}=\left(v_{i}, v_{j}\right) \mu_{j} v_{j}$, where $\mu_{i}$ and $v_{j}$ are fixed nonzero constants.

\section{STABILITY ANALYSIS}

Let us denote $\gamma_{j}$ in $(2.5)$ by $\gamma_{j}^{(n, k)}$ for both MPE and RRE. We say that $s_{n, k}$ is asymptotically stable if

$$
\sup _{n} \sum_{j=0}^{k}\left|r_{j}^{(n, k)}\right|<\infty
$$

Roughly speaking, this means that if errors are introduced in the vectors $x_{m}$, then the error in $s_{n, k}$ stays bounded as $n+\infty$. Since $\sum_{j=0}^{k} r_{j}^{(n, k)}=1$, the most ideal situation is one in which $\gamma_{j}^{(n, k)} \geq 0,0 \leq j \leq k$, for all sufficiently large $n$, so that $\sum_{j=0}^{k}\left|\gamma_{j}^{(n, k)}\right|=\sum_{j=0}^{k} \gamma_{j}^{(n, k)}=1$. 
The following theorem gives the stability properties of both the MPE and RRE when they are applied to vector sequences satisfying the conditions of Theorem 3.1. $s_{n, k}$ denotes either that obtained from MPE or RRE.

Theorem 4.1. For vector sequences satisfying the conditions stated in Theorem 3.1, $s_{n, k}$ is asymptotically stable. Actually, the following are true:

(1) The $r_{q}^{(n, k)}$ satisfy

$$
r_{q}^{(n, k)}=\frac{C_{q}\left(\lambda_{1}, \ldots, \lambda_{k}\right)}{V\left(1, \lambda_{1}, \ldots, \lambda_{k}\right)}[1+o(1)] \quad \text { as } n+\infty \text {, }
$$

where

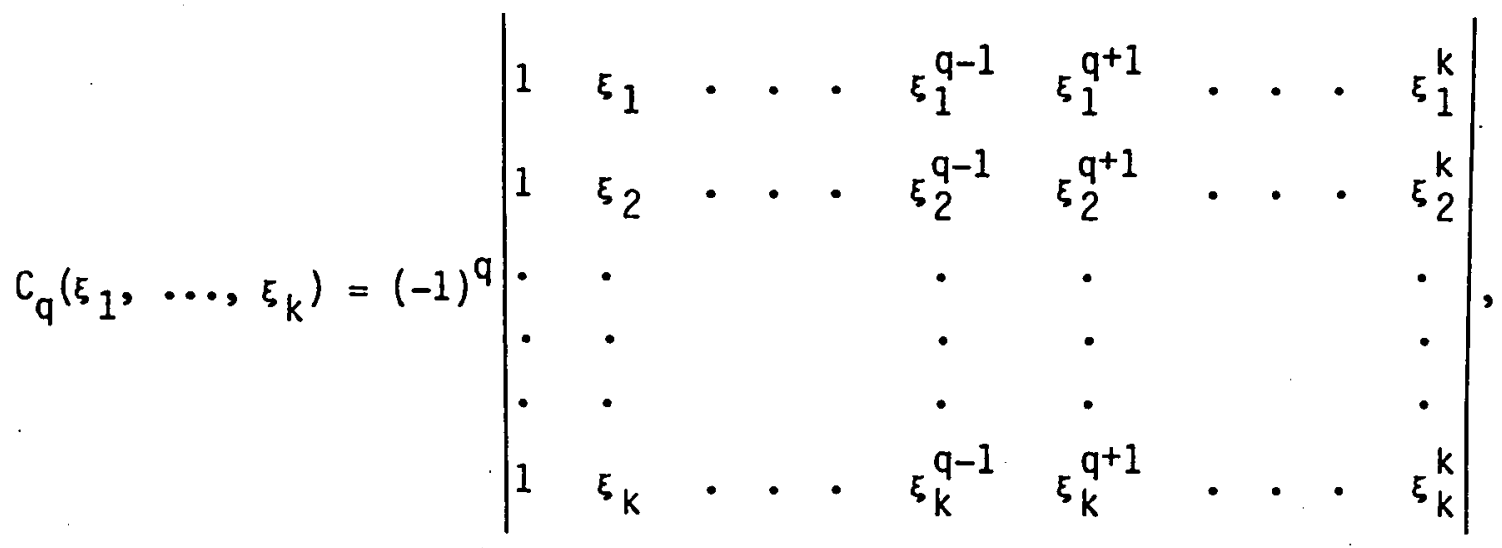

thus

$$
\sum_{q=0}^{k}\left|r_{q}^{(n, k)}\right|=\frac{\sum_{q=0}^{k}\left|C_{q}\left(\lambda_{1}, \ldots, \lambda_{k}\right)\right|}{\left|V\left(1, \lambda_{1}, \ldots, \lambda_{k}\right)\right|}[1+o(1)] \quad \text { as } n+\infty \text {, }
$$

i.e., (4.1) holds.

(2) If $\lambda_{1}, \ldots, \lambda_{k}$ are real and negative, then

$$
r_{q}^{(n, k)}>0,0 \leq q \leq k, \text { for } n \text { sufficientiy large, }
$$

thus 


$$
\sum_{q=0}^{k}\left|r_{q}(n, k)\right|=1+o(1) \quad \text { as } n+\infty
$$

(3) If $\lambda_{1}, \ldots, \lambda_{k}$ are real and positive, then

$$
\gamma_{q}^{(n, k)} \gamma_{q+1}^{(n, k)}<0,0 \leq q \leq k-1 \text {, for } n \text { sufficiently large, }
$$

thus

$$
\sum_{q=0}^{k}\left|\gamma_{q}^{(n, k)}\right|=\left|\prod_{i=1}^{k}\left(\frac{\lambda_{i}+1}{\lambda_{i}-1}\right)\right|[1+o(1)] \quad \text { as } n+\infty \text {. }
$$

(4) For any $\lambda_{1}, \ldots, \lambda_{k}$,

$$
\lim _{n \rightarrow \infty} \sum_{q=0}^{k} \gamma_{q}^{(n, k)}{ }_{\lambda}^{q}=\prod_{i=1}^{k}\left(\frac{\lambda_{i}-\lambda}{\lambda_{i}-1}\right)
$$

Proof. Since $r_{q}^{(n, k)}$ is given by (2.12), and we already know the asymptotic behavior of $D(1, \ldots, 1)$ from Lemma 3.2 , it is sufficient to analyze $N_{q}$ asymptotically. By deleting the first row and the $(q+1)$ st column of the determinant in (3.22), after some manipulation, we have

$$
N_{q} \sim \sum_{i_{1}, j_{1}} \cdots \sum_{i_{k}, j_{k}}\left(\prod_{p=1}^{k} z_{i_{p} j_{p}} \lambda_{i_{p}^{n}}^{n+p-1} \lambda_{j_{p}}^{n}\right) c_{q}\left(\lambda_{j_{1}}, \ldots, \lambda_{j_{k}}\right) .
$$

Observe that $\left(\prod_{p=1}^{k} \lambda_{j}^{n}\right) C_{q}\left(\lambda_{j_{1}}, \ldots, \lambda_{j_{k}}\right)$ is odd under an interchange of any two of the indices $j_{p}$. Consequentiy, 
(4.11) $N_{q} \sim \sum_{1 \leq j_{1}<j_{2}<\ldots<j_{k}}\left(\prod_{p=1}^{k} \lambda_{j_{p}}^{n}\right) c_{q}\left(\lambda_{j_{1}}, \ldots, \lambda_{j_{k}}\right)$

$$
x \sum_{1 \leq i_{1}<i_{2}<\ldots<i_{k}}\left(\prod_{p=1}^{k} \bar{\lambda}_{i_{p}}^{n}\right) \vee\left(\bar{\lambda}_{i_{1}}, \ldots, \bar{\lambda}_{i_{k}}\right) s_{j_{1}, \ldots, j_{k}}^{i_{1}, \ldots, i_{k}}
$$

follows from (4.10) in exactly the same way (3.21) follows from (3.23). Invoking (3.25),

$$
N_{q}=\left|\prod_{p=1}^{k} \lambda_{p}^{n}\right|^{2} v\left(\bar{\lambda}_{1}, \ldots, \bar{\lambda}_{k}\right) c_{q}\left(\lambda_{1}, \ldots, \lambda_{k}\right) s_{1, \ldots, k}^{1, \ldots, k}[1+o(1)]
$$

follows from (4.11) in the same way (3.31) follows from (3.21). Combining (4.12) and (3.31) in (2.12), (4.2) follows. (4.4) follows directly from (4.2). This proves (1).

Note that $V\left(\lambda, \lambda_{1}, \ldots, \lambda_{k}\right)$ is a polynomial of degree $k$ in $\lambda$. From (3.11) and (4.3) we have

$$
V\left(\lambda, \lambda_{1}, \ldots, \lambda_{k}\right)=\sum_{q=0}^{k} c_{q}\left(\lambda_{1}, \ldots, \lambda_{k}\right) \lambda^{q} .
$$

Since $V\left(\lambda, \lambda_{1}, \ldots, \lambda_{k}\right)$ has $\lambda_{1}, \ldots, \lambda_{k}$ as its only zeros, we also have

$$
V\left(\lambda, \lambda_{1}, \ldots, \lambda_{k}\right)=C_{k}\left(\lambda_{1}, \ldots, \lambda_{k}\right) \prod_{i=1}^{k}\left(\lambda-\lambda_{i}\right) .
$$

If $\lambda_{1}, \ldots, \lambda_{k}$ are real and negative, then (4.13) and (4.14) imply that $C_{q}\left(\lambda_{1}, \ldots, \lambda_{k}\right), 0 \leq q \leq k$, are all of the same sign. This, along with (4.2), 
implies (4.5). (4.6) is a direct consequence of (4.5) and (2.11). This proves (2).

If $\lambda_{1}, \ldots, \lambda_{k}$ are real and positive, then (4.13) and (4.14) imply that $C_{q}\left(\lambda_{1}, \ldots, \lambda_{k}\right) C_{q+1}\left(\lambda_{1}, \ldots, \lambda_{k}\right)<0,0 \leq q \leq k-1$. This, along with (4.2), implies (4.7) and

$$
\begin{aligned}
\sum_{q=0}^{k}\left|\gamma_{q}^{(n, k)}\right| & =\frac{\left|\sum_{q=0}^{k}(-1)^{q} c_{q}\left(\lambda_{1}, \ldots, \lambda_{k}\right)\right|}{\left|V\left(1, \lambda_{1}, \ldots, \lambda_{k}\right)\right|}[1+o(1)] \text { as } n+\infty \\
& =\left|\frac{V\left(-1, \lambda_{1}, \ldots, \lambda_{k}\right)}{V\left(1, \lambda_{1}, \ldots, \lambda_{k}\right)}\right|[1+o(1)] \text { as } n+\infty,
\end{aligned}
$$

which, by (3.11), reduces to (4.8). This proves (3).

Multiplying both sides of $(4.2)$ by $\lambda^{q}$, summing over $q$ from 0 to $k$, and finally making use of (4.13) and (3.11), (4.9) follows, thus proving (4).

\section{REFERENCES}

[1] S. Cabay and L. W. Jackson, A polynomial extrapolation method for finding limits and antilimits of vector sequences, SIAM, J. Numer. Anal., $\underline{13}$ (1976), pp. 734-752.

[2] R. P. Eddy, Extrapolating to the limit of a vector sequence, in Information Linkage between Applied Mathematics and Industry, (P.C.C. Wang, ed.), New York; Academic Press, 1979, pp. 387-396.

[3] A. Sidi, W. F. Ford, and D. A. Smith, Acceleration of convergence of vector sequences. NASA TP-2193, 1983. 


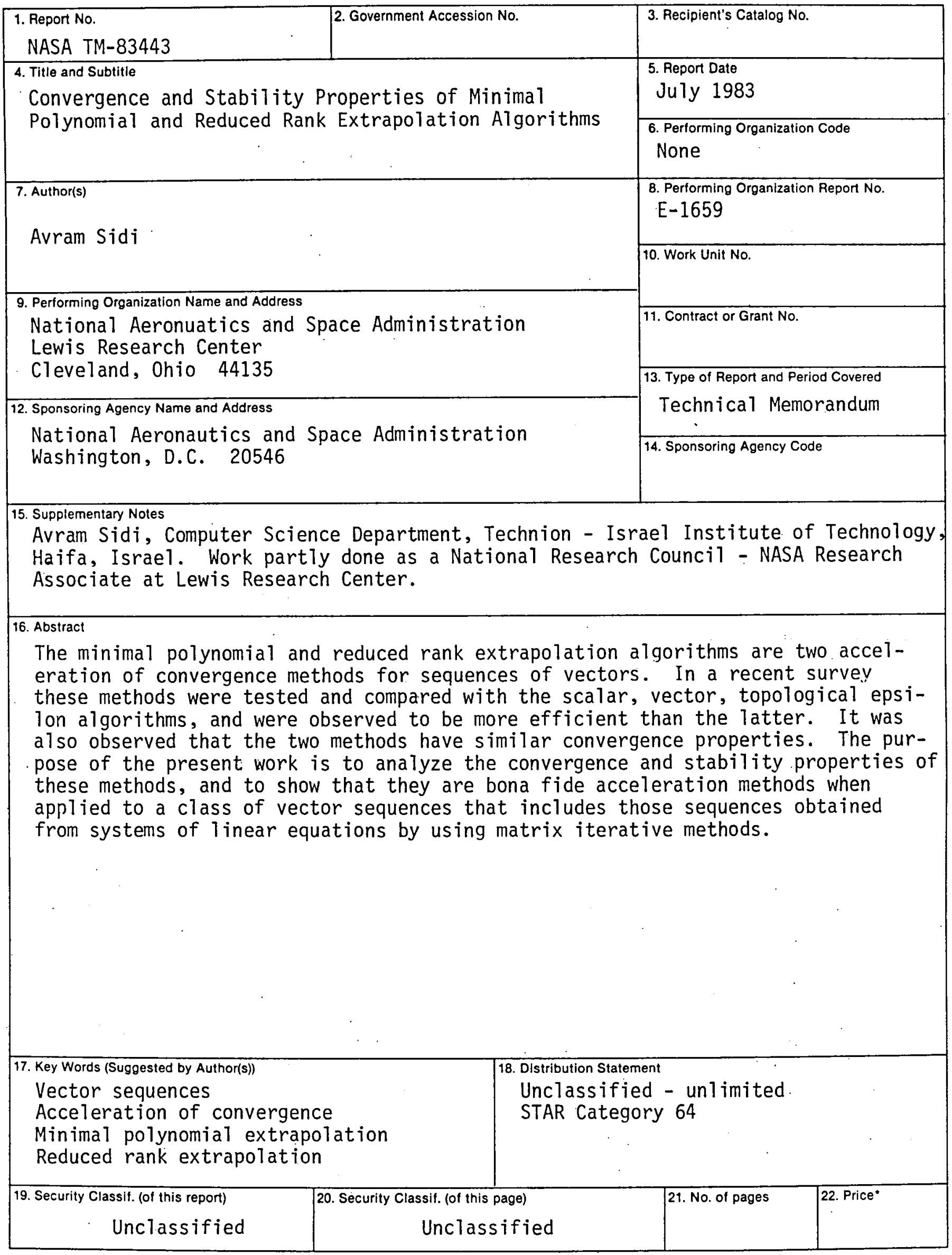

*For sale by the National Technical Information Service, Springfield, Virginia 22161 
National Aeronautics and Space Administration

Washington, D.C.

20546

Official Business

Penaliv for Private Use, $\$ 300$
SPECIAL FOURTH CLASS MAIL BOOK 